Chirurg 2021 · 92:948-954

https://doi.org/10.1007/s00104-020-01331-3

Angenommen: 16. November 2020

Online publiziert: 4. Januar 2021

๑) Der/die Autor(en) 2021
Astrid Bauschke - Annelore Altendorf-Hofmann - Aladdin Ali Deeb ·

Herman Kissler · Hans-Michael Tautenhahn · Utz Settmacher

Klinik für Allgemein-, Viszeral- und Gefäßchirurgie, Universitätsklinikum Jena, Jena, Deutschland

\title{
Chirurgische Therapie von Leber- und Pankreasmetastasen von Nierenzellkarzinomen
}

\section{Hintergrund}

Metastasen des Nierenzellkarzinoms treten bei $20-30 \%$ synchron und bei $50 \%$ der Patienten metachron $[4,26]$ meist in Lunge, Knochen und Leber, aber selten im Pankreas auf $[9,14,17]$. So fanden sich in Autopsiestudien bei $40 \%$ der Patienten Lebermetastasen sowie bei bis zu $12 \%$ der Patienten Pankreasmetastasen [2]. Der Stellenwert der Resektion ggf. in Kombination mit lokal ablativen Verfahren in der Behandlung dieser Metastasen, insbesondere in der oligometastasierten Situation, im Vergleich zu anderen Verfahren (Radio-, Hormon- oder medikamentöse Therapie mit einem Tyrosinkinaseinhibitor) ist bis heute Gegenstand von Diskussionen [25]. Die Europäische Gesellschaft für Urologische Leitlinien stuft die vollständige Resektion von $\mathrm{Me}$ tastasen als probate Therapieoption mit einem prognostischen Vorteil ein [12].

Wir präsentieren die Ergebnisse der chirurgischen Therapie unserer Patienten mit hepatopankreatischen Metastasen oligometastasierter Nierenzellkarzinome in einer retrospektiven Erfassung und diskutieren die Daten mit den Ergebnissen der Literatur.

\section{Material und Methoden}

Die Daten der Patienten stammen aus dem Tumorregister der Klinik für Allgemein-, Viszeral- und Gefäßchirurgie des Universitätsklinikums Jena (Ethikvotum 5073-02/17 Ethikkommission der Universität Jena). Die hypervaskularisierten, malignomsuspekten Raumforderungen wurden schnittbild- gebend mit Computertomographie oder Magnetresonanztomographie diagnostiziert. Die Indikation zur chirurgischen Therapie erfolgte in den letzten Jahren nach interdisziplinärer Befunddiskussion im pankreatohepatobiliären Tumorboard. Alle Resektionen erfolgten parenchymsparend nach Ausdehnung des Befundes über einen offenen (konventionellen) Zugang. Tumorsuspekte lokale Lymphknoten wurden exstirpiert. Leberteilresektionen führen wir ohne PringleManeuver mit dem CUSA („cavitron ultrasonic aspirator“; CUSA ${ }^{\circledR}$, Valleylab, Boulder, Colorado, USA) durch.
Zur statistischen Analyse kam SPSS Software Version 19 zur Anwendung. Unterschiede in der Verteilung von Variablen wurden mit dem Mann-WhitneyU-Test oder mit dem $\chi^{2}$-Test überprüft. Überlebensraten wurden nach KaplanMeier berechnet. Die Signifikanztestung erfolgte mittels Log-Rank-Test.

\section{Ergebnisse}

\section{Gesamtpatientenkollektiv}

Wir haben 18 Patienten mit Pankreas-, 32 Patienten mit Lebermetastasen sowie

\begin{tabular}{|c|c|c|c|}
\hline \multicolumn{2}{|l|}{ Leber } & \multicolumn{2}{|l|}{ Pankreas } \\
\hline Lokalisation & Anzahl & Lokalisation & Anzahl \\
\hline \multicolumn{4}{|c|}{ Tumormanifestationen vor Resektion } \\
\hline Lunge & 5 & Lunge & 5 \\
\hline Nebenniere & 1 & Nebenniere & 1 \\
\hline Lokalrezidiv & 1 & Lokalrezidiv & 1 \\
\hline Bauchwand & 1 & Schilddrüse & 1 \\
\hline Dickdarm & 1 & \multirow[t]{2}{*}{ Hoden } & \multirow[t]{2}{*}{1} \\
\hline Pankreas & 1 & & \\
\hline \multicolumn{4}{|c|}{ Tumormanifestationen zur Resektion } \\
\hline Lunge & 7 & Nebenniere & 2 \\
\hline Nebenniere & 1 & Peripankreatisch & 1 \\
\hline Omentum & 3 & \multirow[t]{3}{*}{ Peritoneum } & \multirow[t]{3}{*}{1} \\
\hline Lokalrezidiv & 3 & & \\
\hline Nicht regionäre Lymphknoten & 2 & & \\
\hline \multicolumn{4}{|c|}{ Tumormanifestationen nach Resektion } \\
\hline Leber & 11 & Leber & 2 \\
\hline Lunge & 5 & Lunge & 4 \\
\hline Hirn & 1 & Skelett & 2 \\
\hline \multirow[t]{2}{*}{ Peritoneum } & \multirow[t]{2}{*}{1} & Restpankreas & 2 \\
\hline & & Weichteile & 1 \\
\hline
\end{tabular}


Tab. 2 Übersicht der R0-Leberresektionen

\begin{tabular}{|c|c|c|c|}
\hline \multicolumn{2}{|l|}{ Merkmal } & \multirow{2}{*}{$\begin{array}{l}\text { Patientenanzahl } \\
23\end{array}$} & \multirow{2}{*}{$\begin{array}{l}\text { Prozent } \\
100\end{array}$} \\
\hline Gesamt & & & \\
\hline Alter & Median (Range) & 63 (45-78) Jahre & - \\
\hline \multirow[t]{2}{*}{ Geschlecht } & Männlich & 15 & 65 \\
\hline & Weiblich & 8 & 35 \\
\hline \multirow[t]{2}{*}{ Leber MTS } & Synchron & 4 & 17 \\
\hline & Metachron & 19 & 83 \\
\hline \multirow[t]{2}{*}{ Anzahl MTS } & Solitär & 10 & 43 \\
\hline & Multipel & 13 & 57 \\
\hline \multicolumn{2}{|l|}{ MTS vorher } & 7 & 30 \\
\hline \multicolumn{2}{|c|}{ Extrahepatischer Tumor } & 8 & 35 \\
\hline \multicolumn{2}{|l|}{ Tumorrezidiv } & 13 & 57 \\
\hline \multirow[t]{3}{*}{ Zweittumoren } & Synchron & 0 & 0 \\
\hline & Vorher & 1 & 4 \\
\hline & Im Verlauf & 5 & 22 \\
\hline \multirow[t]{2}{*}{ Leberresektion } & $<3$ Segmente & 7 & 30 \\
\hline & $\geq 3$ Segmente & 16 & 70 \\
\hline Nachbeobachtung & Median (Range) & 15 (0-244) Monate & - \\
\hline \multicolumn{2}{|c|}{ Komplikationen Clavien $\geq$ III (30 Tage) } & 3 & 13 \\
\hline \multicolumn{2}{|l|}{ Todesfälle (30Tage) } & 1 & 4 \\
\hline
\end{tabular}

Tab. 3 Übersicht der R0-Pankreasresektionen

\begin{tabular}{|c|c|c|c|}
\hline \multicolumn{2}{|l|}{ Merkmal } & \multirow{2}{*}{$\begin{array}{l}\text { Patientenanzahl } \\
16\end{array}$} & \multirow{2}{*}{$\begin{array}{l}\text { Prozent } \\
100\end{array}$} \\
\hline Gesamt & & & \\
\hline Alter & Median (Range) & 71 (36-79) Jahre & - \\
\hline \multirow[t]{2}{*}{ Geschlecht } & Männlich & 9 & 56 \\
\hline & Weiblich & 7 & 44 \\
\hline \multirow[t]{2}{*}{ Leber MTS } & Synchron & 0 & 0 \\
\hline & Metachron & 16 & 100 \\
\hline \multirow[t]{2}{*}{ Anzahl MTS } & Solitär & 13 & 81 \\
\hline & Multipel & 3 & 19 \\
\hline \multicolumn{2}{|l|}{ MTS vorher } & 8 & 50 \\
\hline \multicolumn{2}{|l|}{ Extrapankreatischer Tumor } & 3 & 19 \\
\hline \multicolumn{2}{|l|}{ Tumorrezidiv } & 9 & 56 \\
\hline \multirow[t]{3}{*}{ Zweittumoren } & Synchron & 2 & 13 \\
\hline & Vorher & 3 & 19 \\
\hline & Im Verlauf & 1 & 6 \\
\hline Nachbeobachtung (Monate) & Median (Range) & $60(2-156)$ & - \\
\hline \multicolumn{2}{|l|}{ Pankreatektomie } & 4 & 25 \\
\hline \multicolumn{2}{|l|}{ Pankreaskopfresektion } & 3 & 19 \\
\hline \multicolumn{2}{|l|}{ Pankreaslinksresektion } & 9 & 56 \\
\hline \multicolumn{2}{|c|}{ Komplikationen Clavien $\geq$ III (30 Tage) } & 5 & 31 \\
\hline \multicolumn{2}{|l|}{ Todesfälle (30 Tage) } & 0 & 0 \\
\hline \multicolumn{4}{|l|}{ MTS Metastase } \\
\hline
\end{tabular}

einen weiteren Patienten mit Metastasen in Leber und Pankreas im Abstand von 2 Jahren operiert. Alle Resektionen im Zeitraum von 1995 bis 2018 erfolgten in kurativer Intention.

Die applizierte Systemtherapie unterlag einem Wandel. Die Tumormanifestationen vor, zum Zeitpunkt und nach der Leber- oder Pankreasresektion aller 52 Fälle sind in $\bullet$ Tab. 1 aufgeführt.

Die 5-Jahres-Überlebensrate aller Patienten nach Leberresektion war statistisch signifikant geringer (38 $\pm 9 \%$, mediane Überlebenszeit 34 Monate) als nach Pankreasresektion (69 $\pm 11 \%$, mediane Überlebenszeit 69 Monaten; $p=0,017) .21$ Patienten haben bislang die Metastasenentfernung länger als 5 Jahre überlebt, 4 Patienten länger als 10 Jahre. Die mediane Nachbeobachtungszeit der verstorbenen Patienten beträgt 34 (0-244 Monate), die der noch lebenden 69 (12-174) Monate. Am Ende der Beobachtung waren 37 der 51 Patienten verstorben.

Die 23 Patienten mit einer R0-Leberresektion hatten ein statistisch signifikant schlechteres Langzeitüberleben als die 16 Patienten mit R0-Pankreasresektion $(p=0,026)$. Die 5-Jahres-Überlebensrate nach Leberresektion war $37 \pm 10 \%$ (mediane Überlebenszeit 34 Monate), nach Pankreasresektion $68 \pm 13 \%$ (mediane Überlebenszeit 65 Monate; • Abb. 1).

\section{Leber}

Von den 33 wegen Lebermetastasen therapierten Patienten erhielten 31 (94\%) Patienten eine Resektion sowie 2 eine Kombination von Resektion und Ablation. Es erfolgte bei 24 (73\%) Patienten eine Major- bzw. bei 9 Patienten (27\%) eine Minorresektion. In der pathologischen Analyse wurde eine R0-, R1- bzw. R2-Resektion bei 23 (70\%), 3 (9\%) bzw. 7 (21\%) Patienten beschrieben.

Die Morbidiät (Clavien $\geq$ III) sowie Mortalität der Leberresektionen unserer Studie betrugen $18 \%$ sowie $6 \%$. 2 Patienten sind innerhalb der ersten $30 \mathrm{Ta}$ ge postoperativ verstorben. Fünf der leberresezierten Patienten wurden adjuvant mit Sunitinib therapiert. Im metastasierten Stadium mehrere Jahre nach Leberresektion wurden weitere 5 Pati- 
enten mit Sunitinib sowie jeweils $2 \mathrm{~Pa}$ tienten chemo- bzw. immuntherapiert. Vor den 33 Lebereingriffen wurden bei 10 Patienten (30\%) andere Rezidive entfernt. Acht der 23 Patienten mit R0-Leberresektion wurden mehrfach an der Leber operiert. Eine Übersicht über die R0-resezierten Patienten zeigt • Tab. 2. Bei R0-Leberresektionen erwiesen sich in der univariaten Analyse ein Intervall von <24 Monaten zwischen Nephrektomie und Leberresektion sowie multiple Metastasen als negative Prognosefaktoren (•Abb. 2). Extrahepatische Tumorabsiedelungen zum Zeitpunkt des Eingriffs, der Prognose-Score nach Adam et al. [1] sowie vorherige Metastasen hatten dagegen keinen statistisch signifikanten Einfluss auf das Überleben. Die kumulative Rezidivrate nach 5 Jahren betrug bei der Leber $62 \%$ (• Abb. 3).

\section{Pankreas}

Von den 19 Patienten mit Pankreasmetastasen wurde bei 3 Patienten eine Pankreaskopfresektion, bei 4 Patienten eine Pankreatektomie sowie bei den übrigen 12 Patienten eine Pankreaslinksresektion durchgeführt. Drei pankreasresezierte Patienten wurden adjuvant mit Sunitinib therapiert. In der pathologischen Untersuchung wurde eine R0- bzw. R1Resektion bei 16 (84\%) bzw. 3 (16\%) Patienten beschrieben. Kein Patient war postoperativ verstorben, die Morbidität (Clavien $\geq$ III) betrug $31 \%$. Eine Übersicht über die R0-resezierten Patienten zeigt • Tab. 3. Die kumulative Rezidivrate nach 5 Jahren betrug beim Pankreas $43 \%$ (• Abb. 3 ).

\section{Diskussion}

Der Stellenwert der chirurgischen Therapie oligometastasierter Tumorerkrankungen wird aktuell diskutiert. Die vorliegenden Studien haben einen unterschiedlichen Case-Mix insbesondere auch im Hinblick auf potenzielle Prognosefaktoren (- Tab. 4). So unterlag die Systemtherapie im Untersuchungszeitraum einem zeitlichen Wandel. Dies erschwert die Wertung der Therapieergebnisse.

Chirurg 2021 · 92:948-954 https://doi.org/10.1007/s00104-020-01331-3

(c) Der/die Autor(en) 2021

A. Bauschke · A. Altendorf-Hofmann · A. Ali Deeb · H. Kissler · H.-M. Tautenhahn · U. Settmacher

\section{Chirurgische Therapie von Leber- und Pankreasmetastasen von Nierenzellkarzinomen}

\section{Zusammenfassung}

Hintergrund. Der Stellenwert der chirurgischen Therapie hepatopankreatischer Metastasen des oligometastasierten Nierenzellkarzinoms ist Gegenstand der aktuellen Diskussion.

Material und Methoden. Wir berichten über 51 Patienten, von denen 33 wegen Lebermetastasen und 19 wegen Pankreasmetastasen im Zeitraum von 1995 bis 2018 operiert wurden.

Ergebnisse. Die 5-Jahres-Überlebensrate aller Patienten nach Leberteilresektion war statistisch signifikant geringer $(38 \%$, mediane Überlebenszeit 34 Monate) als nach Pankreasresektion (69\%, mediane Überlebenszeit 69 Monaten; $p=0,017) .21$ Patienten haben bislang die Metastasenentfernung länger als 5 Jahr überlebt, 4 Patienten länger als 10 Jahre. Bei den R0-resezierten Patienten wurden Rezidive in 13 Fällen nach Leber- und 9 Fällen nach Pankreasresektion beobachtet. Die kumulative Rezidivrate nach 5 Jahren betrug bei der Leber $38 \%$ und beim Pankreas 57\%. Bei R0-Leberteilresektionen erwiesen sich ein Intervall von $<24$ Monaten zwischen Nephrektomie und Leberresektion sowie multiple Metastasen als negative Prognosefaktoren.

Diskussion. Unsere Ergebnisse gestatten eine aktive chirurgische Strategie in der Behandlung hepatopankreatischer Metastasen oligometastasierter Nierenzellkarzinome, insbesondere bei kompletter Resektion solitärer, metachroner Metastasen. Wiederholte Eingriffe bei komplett resektablen Metastasen führen zu langen tumorfreien Intervallen und tragen damit zu guten Langzeitergebnissen bei.

\section{Schlüsselwörter}

Hepatopankreatische Metastasen · Chirurgie . Morbidität · Mortalität · Risikofaktoren

\section{Surgical treatment of hepato-pancreatic metastases from renal cell carcinoma}

\section{Abstract}

Background. The role of surgical treatment of hepato-pancreatic metastases from renal cell carcinoma is still under discussion.

Material and methods. We report about 52 patients of whom 33 underwent surgery for liver metastases and 19 for pancreatic metastases from 1995 to 2018.

Results. The 5-year survival rate of all patients with partial liver resection was statistically significantly lower $(38 \%$, median survival time 34 months) than with pancreas resection (69\%, median survival time 69 months, $p=0.017$ ). Of the patients 21 survived the resection of metastases longer than 5 years and 4 patients longer than 10 years. In R0 resected patients, recurrences were observed in 13 cases after liver resection and in 9 cases after pancreas resection. The cumulative recurrence rate after 5 years was $38 \%$ for the liver and 57\% for the pancreas. In R0 partial liver resections, an interval $<24$ months between nephrectomy and liver resection as well as multiple metastases were negative prognostic factors.

Conclusion. In spite of high recurrence rates, surgical treatment for hepato-pancreatic metastases from renal cell carcinoma yielded very good long-term results, in particular with complete resection of solitary metachronous metastases. Repeated surgery for completely resectable metastases, resulted in long tumor-free intervals and thus contributed to good long-term results.

\section{Keywords}

Hepato-pancreatic metastases - Surgery · Morbidity $\cdot$ Mortality $\cdot$ Risk factors

\section{Leber}

Mit Senkung der perioperativen Morbidität und Mortalität (• Tab.4) wird über die Indikationsstellung der Metastasenchirurgie nichtkolorektaler Tumoren an der Leber und die sinnvolle Auswahl der Patienten nach Art des Primarius und der Tumorlast diskutiert [1]. Die Analyse ist infolge der langen Krankheitsverläufe sowie der Anwendung verschiedener Systemtherapien komplex. Das tumorfreie 


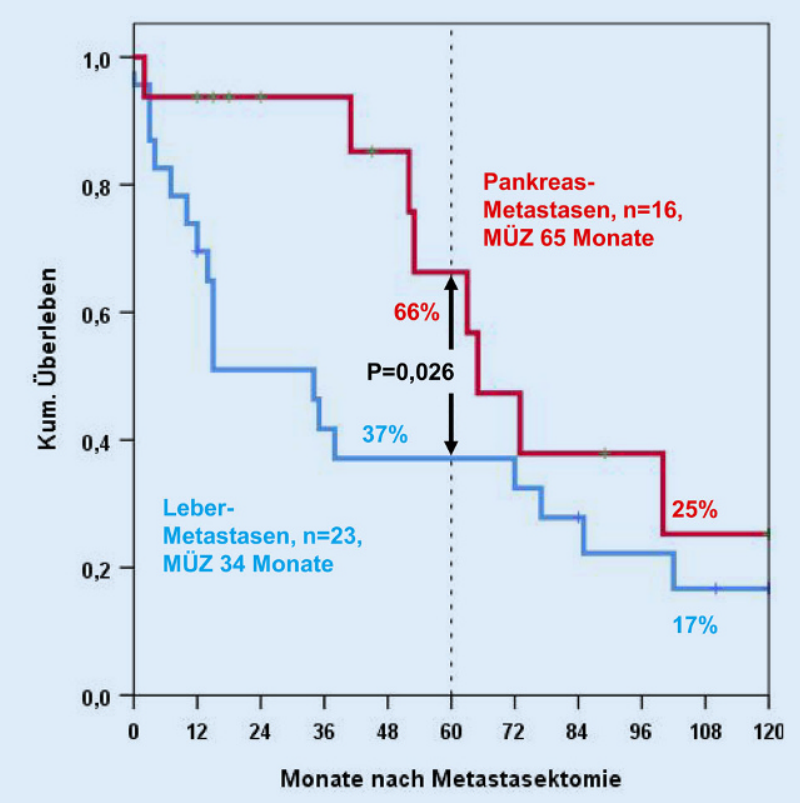

Intervall bis zum Auftreten von Lebermetastasen ist mit etwa 5 Jahren sehr viel kürzer als das tumorfreie Intervall bis zum Auftreten von Pankreasmetastasen mit etwa 10 Jahren (• Tab. 4). In der untersuchten Literatur variierte der Anteil von Patienten mit Metastasen vor der Leberresektion zwischen 0 und $37 \%$, der Anteil mit synchronen Metastasen zum Zeitpunkt der Leberresektion zwischen 8 und $35 \%$, der Anteil solitärer Tumoren zwischen 26 und $78 \%$ sowie der Anteil der R0-Resektionen zwischen 76 und $100 \%$ (• Tab. 4). Der Vergleich der Ergebnisse ist schwierig, da der Einfluss der dargestellten Parameter auf die Prognose unklar ist. In der größten Untersuchung von Adam et al. wird für 85 Patienten mit Lebermetastasen eines Nierenkarzinoms eine 5-Jahres-Überlebensrate von $38 \%$ angegeben, jedoch fehlen die Angaben zu prognostischen Parametern [1].

Das Langzeitüberleben unserer R0leberteilresezierten Patienten wurde in der univariaten Analyse statistisch signifikant von der Anzahl der Lebermetastasen sowie dem Intervall zwischen $\mathrm{Ne}$ phrektomie und Leberresektion beeinflusst.

In der Literatur beeinflussten die R-Klassifikation des Lebereingriffs [10, $16,23]$, das Grading [20], das Intervall zwischen Nephrektomie und Diagnose
Abb. $1<$ Beobachtete Überlebensrate nach R0-Metastasektomie. MÜZmediane Überlebenszeit der Literatur zwischen 24 und 142 Monaten (• Tab. 4).

Auffallend sind die guten Langzeitergebnisse von Staehler et al. [20]. In dieser Studie wurden jedoch Patienten mit extrahepatischem Tumor ausgeschlossen und die Mehrzahl der Patienten adjuvant therapiert. Die Autoren sahen einen Überlebensvorteil bei den Patienten mit Metastasektomie ( $p=0,087 ;[20])$. Auch Pinotti et al. bestätigten im Rahmen eines systematischen Reviews von $378 \mathrm{~Pa}$ tienten mit Lebermetastasen von Nierenzellkarzinomen den prognostischen Vorteil einer chirurgischen Therapie auf das Überleben. Die Einbindung der Patienten in multimodale Therapiekonzepte ermöglicht die Identifikation der Patienten, welche im metastasierten Tumorstadium einen realen Benefit von der Chirurgie haben, wobei die Reihenfolge von Target-Therapie und Chirurgie noch nicht abschließend geklärt ist [15].

der Metastase [3, 5, 10, 11, 16, 20, 23], andere Tumormanifestationen vor der Lebermetastase [9], der Nachweis eines extrahepatischen Tumors zum Zeitpunkt der Leberresektion [5, 9], die Metastasengröße [10], der Performance-Status des Patienten sowie eine Systemtherapie [10] die Prognose nach Leberteilresektion.

Das zeitliche Auftreten der Metastasen - synchron, Frühmetastasen sowie Spätmetastasen - wird unterschiedlich definiert. Nur Hatzaras et al. [9] sahen keinen statistisch signifikanten Einfluss des Intervalls zwischen Resektion des Primarius und der Metastasektomie auf das Überleben (•Tab. 4).

In den Arbeiten von Hatzaras et al., Hau et al., Beetz et al. sowie Staehler et al. wurden die wegen Lebermetastasen von Nierenzellkarzinomen operierten Patienten zwischen 37 und $80 \%$ mit Immun- bzw. Chemotherapie behandelt $[5,9,10,20]$. Beetz et al. und Hau et al. sahen ein statistisch signifikant besseres Überleben bei den mit Tyrosinkinaseinhibitor (Sunitinib) therapierten Patienten $(p=0,038 ;[5,10])$. Hau et al. sahen außerdem bei einem ECOG(Eastern Cooperative Oncology Group)-Status $>1$ sowie synchronen Lebermetastasen einen negativen Einfluss auf das Überleben [10]. Die mediane Überlebenszeit variierte in

\section{Pankreas}

Metastasenchirurgie des Pankreas ist deutlich seltener. Pankreasresektionen wegen Metastasen erfolgen bei ca. 2-5\% aller Pankreasresektionen [4]. Der Anteil von Metastasen von Nierenzellkarzinomen beträgt $50-60 \%$ [19]. Eine niedrige perioperative Mortalität und Morbidität sind dafür die unabdingbare Voraussetzung (•Tab. 4). In der Literatur beeinflussten eine Gefäßinvasion [24], eine Tumormanifestation vor der Pankreasmetastase [18], der Nachweis eines extrapankreatischen Tumors [22], das Intervall zwischen Nephrektomie und Pankreasresektion [22] sowie eine symptomatische Erkrankung [24] die Prognose nach Pankreasresektion.

Vorangegangene Metastasenchirurgie war kein negativer Prognosefaktor [22, 30]. Neben dem perioperativen Risiko muss der Langzeiterfolg im Vergleich zur Systemtherapie sowie die Option anderer wirksamer Therapien in Kombination mit einem chirurgischen Eingriff berücksichtigt werden [21]. In der Studie von Grassi et al. überlebten die Patienten mit operativer Therapie länger als die systemisch therapierten Patienten (106 bzw. 59 Monate; [8]). In der retrospektiven multizentrischen Studie von 


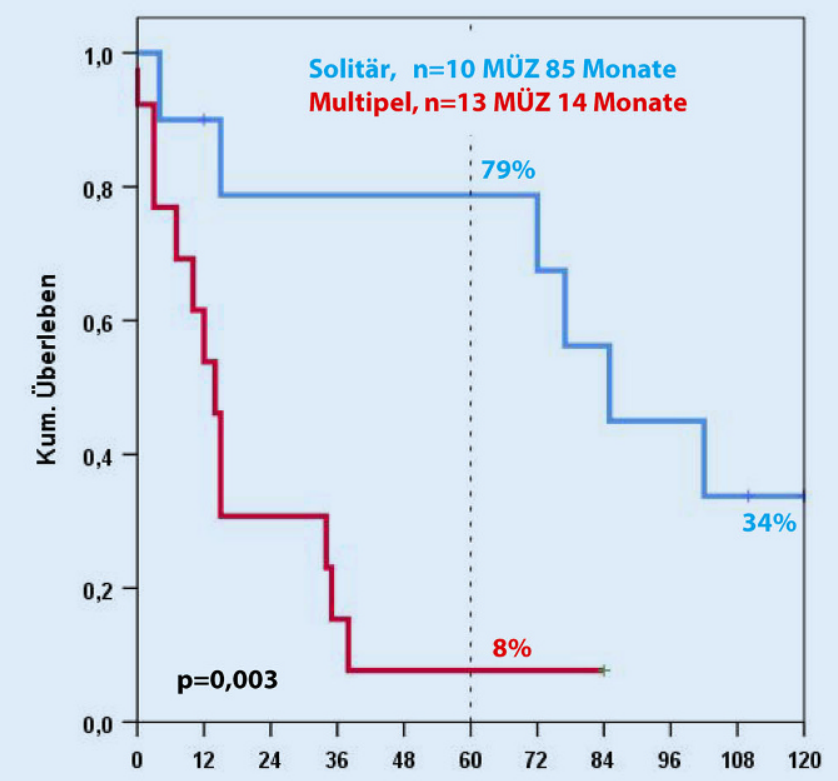

a

Monate nach Metastasektomie

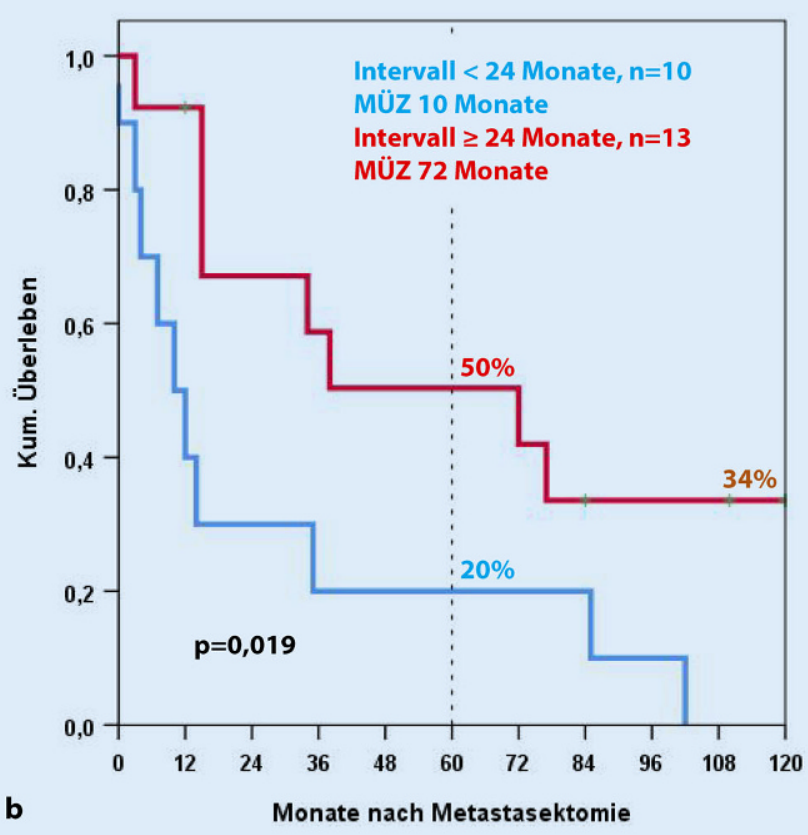

Abb. $2 \Delta$ Beobachtetes Überleben nach R0-Leberresektion in Abhängigkeit von der Anzahl der Lebermetastasen (a) und des Intervalls Primärtherapie - Leberteilresektion (b), MÜZmediane Überlebenszeit

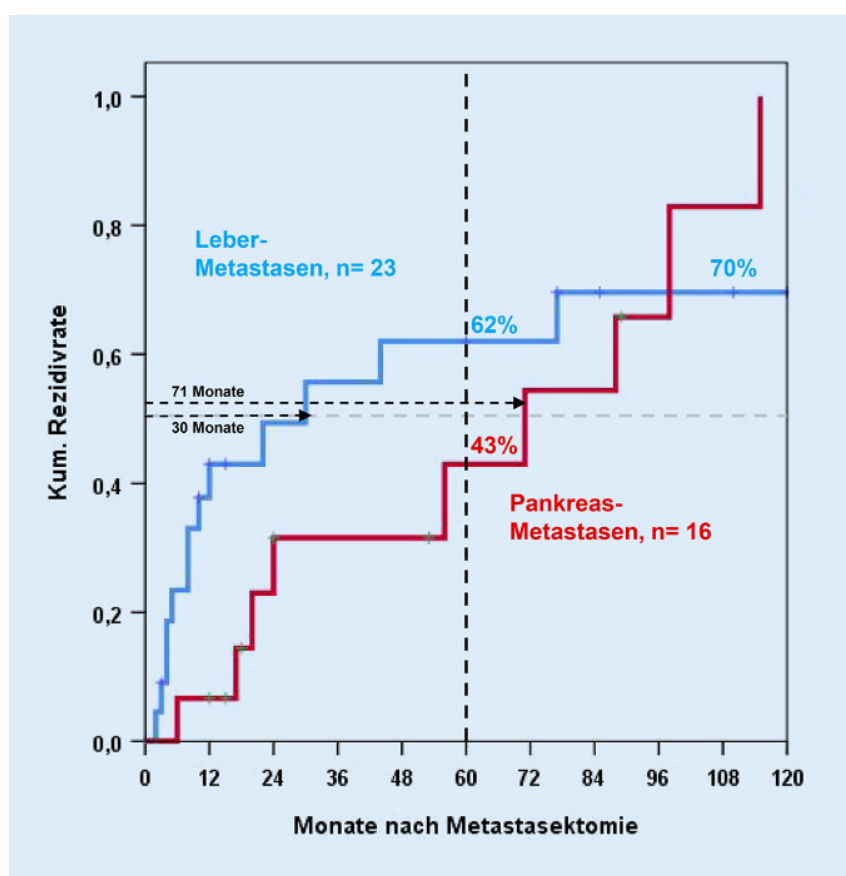

Abb. $3<$ Kumulierte Rezidivraten nach R0-Leberresektion bzw. R0-Pankreasresektion
Santoni et al. fand sich kein statistisch signifikanter Unterschied im medianen Überleben (Operation vs. Tyrosinkinaseinhibitor 103 vs. 86 Monate). Jedoch muss man die chemotherapiebedingten Nebenwirkungen dem perioperativen Risiko gegenüberstellen [17]. Lediglich in der Arbeit von Schwarz et al. [18] wur- den $5 \%$ der wegen Pankreasmetastasen operierten Patienten adjuvant systemisch therapiert.

Randomisierte prospektive Studien zum Stellenwert der Metastasenresektion resektabler Pankreastumoren sind bei dieser seltenen Tumorentität schwer vorstellbar.

\section{Stellenwert der kompletten Metastasektomie}

Es existieren bisher keine randomisierten Studien zum Stellenwert der kompletten Metastasektomie beim metastasierten Nierenzellkarzinom. In dem systematischen Review von 16 Studien von Dabestani et al. [6] sowie dem systematischen Review und der Metaanalyse (2267 Patienten) von Zaid et al. [29] profitierten die Patienten von einer kompletten Metastasektomie mit einem verbesserten Überleben sowie einer Symptomkontrolle. Allerdings betrachten die Autoren überwiegend Lungen-, Knochenund Hirnmetastasen, Patienten mit hepatopankreatische Metastasen sind sehr selten Auch wenn die Auswahl keine Aussage für einzelne Tumorlokalisationen zulässt, so zeigt sich ein allgemeiner Trend für die chirurgische Therapie. Für metachrone hepatopankreatische Metastasen bei Patienten in gutem Allgemeinzustand wird unter Abwägung der perioperativen Risiken die operative Therapie, ggf. in Kombination mit lokal ablativen Therapien, empfohlen. Dagegen sollte bei Patienten mit eingeschränktem Allgemeinzustand sowie kurzem Intervall zwischen Nephrektomie und Mani- 


\begin{tabular}{|c|c|c|c|c|c|c|c|c|c|c|c|c|}
\hline & Jahr & Pat & $\begin{array}{l}\text { MTS } \\
\text { vorher } \\
(\%)\end{array}$ & $\begin{array}{l}\text { MTS syn- } \\
\text { chron } \\
(\%)\end{array}$ & $\begin{array}{l}\text { Tumorfreies } \\
\text { Intervall }\end{array}$ & $\begin{array}{l}\text { EHT/EPT } \\
\text { (\%) }\end{array}$ & $\begin{array}{l}\text { Solitär } \\
\text { (\%) }\end{array}$ & $\begin{array}{l}\text { R0-rese- } \\
\text { ziert } \\
(\%)\end{array}$ & $\begin{array}{l}\text { Morta- } \\
\text { lität } \\
(\%)\end{array}$ & $\begin{array}{l}\text { Morbidität } \\
\text { Clavien } \geq \text { III } \\
\text { (\%) }\end{array}$ & $\begin{array}{l}\text { MÜZ } \\
\text { (Mo) }\end{array}$ & $\begin{array}{l}\text { 5-JÜR } \\
\text { (\%) }\end{array}$ \\
\hline \multicolumn{13}{|l|}{ Leber } \\
\hline Aloia et al. [3] & 2006 & 19 & 37 & 26 & $53 \mathrm{Mo}$ & 37 & 42 & 89 & 5,3 & 32 & 36 & 26 \\
\hline $\begin{array}{l}\text { Thelen et al. } \\
\text { [23] }\end{array}$ & 2007 & 31 & 19 & 19 & $\mathrm{kA}$ & 10 & $\mathrm{kA}$ & 77 & 3,2 & 12,9 & 48 & 39 \\
\hline $\begin{array}{l}\text { Staehler et al. } \\
\text { [20] }\end{array}$ & 2010 & 68 & $\mathrm{kA}$ & 28 & $\mathrm{kA}$ & 0 & 38 & $\mathrm{kA}$ & 0 & 20 & 142 & 62 \\
\hline Ruys et al. ${ }^{a}[16]$ & 2011 & $33^{c}$ & $\mathrm{kA}$ & 35 & $\begin{array}{l}50 \mathrm{Mo} \\
(7-360)\end{array}$ & 33 & 66 & 76 & 0 & 18 & 33 & 43 \\
\hline $\begin{array}{l}\text { Hatzaras et al. } \\
\text { [9] }\end{array}$ & 2012 & $43^{f}$ & 0 & 21 & $\begin{array}{l}17 \text { Mo } \\
(2-189)\end{array}$ & 33 & 26 & 95 & 2 & $23 \%$ & $>60$ & 62 \\
\hline $\begin{array}{l}\text { Langan et al. }{ }^{a} \\
\text { [11] }\end{array}$ & 2012 & $18^{d}$ & kA & 28 & $\begin{array}{l}54 \mathrm{Mo} \\
(12-144)\end{array}$ & 22 & 78 & 100 & 0 & 30 & 24 & 34 \\
\hline Hau et al. [10] & 2016 & $35^{9}$ & kA & 17 & $\begin{array}{l}15 \mathrm{Mo} \\
(3-39)\end{array}$ & 20 & $\mathrm{kA}$ & 86 & 0 & $20 \%$ & $\mathrm{kA}$ & 51 \\
\hline Beetz et al. [5] & 2020 & 40 & $\mathrm{kA}$ & 8 & $\begin{array}{l}44 \mathrm{Mo} \\
(3-278)\end{array}$ & 35 & $\mathrm{kA}$ & 92 & 2 & 23 & 38 & $\approx 38$ \\
\hline Eigene Daten & 2020 & $33^{e}$ & $30 \%$ & 15 & $\begin{array}{l}31 \mathrm{Mo} \\
(9-183)\end{array}$ & 48 & 36 & 70 & 6 & 18 & 34 & 41 \\
\hline \multicolumn{13}{|l|}{ Pankreas } \\
\hline Zerbi et al. [30] & 2008 & 23 & 23 & 0 & 8 Jahre & 48 & 65 & $\mathrm{kA}$ & 0 & 48 & $\mathrm{kA}$ & 88 \\
\hline $\begin{array}{l}\text { Tanis et al. }{ }^{\text {b }} \\
\text { [22] }\end{array}$ & 2009 & 321 & 10 & 8 & 10 Jahre & 9 & 65 & $\mathrm{kA}$ & 3 & $\mathrm{kA}$ & 69 & 67 \\
\hline $\begin{array}{l}\text { Yazbek u. } \\
\text { Gayet [27] }\end{array}$ & 2012 & 11 & 18 & 9 & $\approx 11$ Jahre & 0 & 100 & 90 & 1 & 55 & 78 & $\mathrm{kA}$ \\
\hline $\begin{array}{l}\text { Schwarz et al. } \\
\text { [18] }\end{array}$ & 2014 & 62 & 13 & 3 & 10 Jahre & 10 & 63 & $\mathrm{kA}$ & 6 & $\mathrm{kA}$ & $\mathrm{kA}$ & 63 \\
\hline $\begin{array}{l}\text { Tosoian et al. } \\
\text { [24] }\end{array}$ & 2014 & 42 & kA & 10 & 11 Jahre & 19 & $\mathrm{kA}$ & 88 & 5 & 19 & $\mathrm{kA}$ & 50 \\
\hline $\begin{array}{l}\text { Fikatas et al. } \\
\text { [7] }\end{array}$ & 2016 & 19 & $\mathrm{kA}$ & 0 & $\approx 10$ Jahre & 22 & $\mathrm{kA}$ & $\mathrm{kA}$ & 0 & 21 & $\mathrm{kA}$ & 71 \\
\hline $\begin{array}{l}\text { Madkhali et al. } \\
\text { [13] }\end{array}$ & 2018 & 17 & $\mathrm{kA}$ & 35 & $\mathrm{kA}$ & 35 & $\mathrm{kA}$ & 100 & 0 & n.s. & $\mathrm{kA}$ & 47 \\
\hline Eigene Daten & 2020 & 19 & 47 & 5 & 13 Jahre & 21 & 74 & 84 & 0 & 31 & 69 & 73 \\
\hline \multicolumn{13}{|c|}{$\begin{array}{l}\text { MTS Metastasen, Mo Monate, EHT extrahepatischer Tumor bei Leberresektion, EPT extrapa } \\
\text { benszeit nach Metastasektomie, } K A \text { keine Angabe, JÜR Jahresüberlebensrate } \\
\text { a'Multizentrische Studie } \\
\text { beview } \\
\text { '4 Patienten Radiofrequenzablation } \\
{ }^{\text {d}} 9 \text { Patienten Radiofrequenzablation } \\
\text { '2 Patienten Resektion + Radiofrequenzablation, } 1 \text { Patient selektive interne Radiotherapie } \\
\text { f7 Patienten Radiofrequenzablation } \\
{ }^{9} 2 \text { Patienten Radiofrequenzablation }\end{array}$} \\
\hline
\end{tabular}

festation der Metastase eher eine Systemtherapie erwogen werden [6]. In der Ära der Target-Therapie beobachteten Yu et al. ein medianes Überleben von 52, 16 bzw. 22 Monaten bei kompletter, inkompletter Metastasektomie bzw. TargetTherapie (mTOR-Inhibitor, Multikinaseinhibitor). Die Patienten mit einer kompletten Metastasektomie hatten ein statistisch signifikant besseres Überleben als die Nichtoperierten. Das Überleben bei inkompletter Metastasektomie bzw. Target-Therapie war nicht statistisch signifikant unterschiedlich [28].

\section{Schlussbetrachtung}

Patienten mit Pankreasmetastasen hatten ein besseres Überleben als Patienten mit Lebermetastasen eines Nieren- zellkarzinoms. In unserer Studie konnte durch einen chirurgischen Eingriff, ggf. in Kombination mit einem lokal ablativen Verfahren, der Krankheitsverlauf sowie das Überleben der Patienten mit hepatopankreatischen Metastasen von Nierenzellkarzinomen positiv beeinflusst werden. 


\section{Fazit für die Praxis}

\section{- Aus unserer Studie resultieren Hin- weise, dass die Prognose von Pa- tienten mit hepatopankreatischen Metastasen oligometastasierter Nierenzellkarzinome durch Metasta- sektomie verbessert werden kann. \\ - Inwieweit neoadjuvante/adjuvante Systemtherapien die Überlebens- und Rezidivraten verbessern können, bleibt abzuwarten. \\ - Es wären prospektive Studien zur Eva- luierung des Stellenwertes der neo- sowie adjuvanten Systemtherapie wünschenswert.}

\section{Korrespondenzadresse}

PD Dr. med. Astrid Bauschke

Klinik für Allgemein-, Viszeral- und Gefäßchirurgie, Universitätsklinikum Jena Erlanger Allee 101, 07740 Jena, Deutschland astrid.bauschke@med.uni-jena.de

Funding. Open Access funding enabled and organized by Projekt DEAL.

\section{Einhaltung ethischer Richtlinien}

Interessenkonflikt. A. Bauschke, A. Altendorf-Hofmann, A. Ali Deeb, H. Kissler, H.-M. Tautenhahn und $U$. Settmacher geben an, dass kein Interessenkonflikt besteht.

Für diesen Beitrag wurden von den Autoren keine Studien an Menschen oder Tieren durchgeführt. Für die aufgeführten Studien gelten die jeweils dort angegebenen ethischen Richtlinien.

Open Access. Dieser Artikel wird unter der Creative Commons Namensnennung 4.0 International Lizenz veröffentlicht, welche die Nutzung, Vervielfältigung Bearbeitung, Verbreitung und Wiedergabe in jeglichem Medium und Format erlaubt, sofern Sie den/die ursprünglichen Autor(en) und die Quelle ordnungsgemäß nennen, einen Link zur Creative Commons Lizenz beifügen und angeben, ob Änderungen vorgenommen wurden.

Die in diesem Artikel enthaltenen Bilder und sonstiges Drittmaterial unterliegen ebenfalls der genannten Creative Commons Lizenz, sofern sich aus der Abbildungslegende nichts anderes ergibt. Sofern das betreffende Material nicht unter der genannten Creative Commons Lizenz steht und die betreffende Handlung nicht nach gesetzlichen Vorschriften erlaubt ist, ist für die oben aufgeführten Weiterverwendungen des Materials die Einwilligung des jeweiligen Rechteinhabers einzuholen.
Weitere Details zur Lizenz entnehmen Sie bitte der Lizenzinformation auf http://creativecommons.org/ licenses/by/4.0/deed.de.

\section{Literatur}

1. Adam R, Chiche L, Aloia T et al (2006) Hepatic resection for noncolorectal nonendocrine liver metastases: analysis of 1,452 patients and development of a prognostic model. Ann Surg 244:524-535

2. Adsay NV, Andea A, Basturk O et al (2004) Secondary tumors of the pancreas: an analysis of a surgical and autopsy database and review of the literature. Virchows Arch 444:527-535

3. Aloia TA, Adam R, Azoulay D et al (2006) Outcome following hepatic resection of metastatic renal tumors: the Paul Brousse Hospital experience. HPB (Oxford) 8:100-105

4. Ballarin R, Spaggiari M, Cautero N et al (2011) Pancreatic metastases from renal cell carcinoma: the state of the art. World J Gastroenterol 17:4747-4756

5. Beetz O, Soffker R, Cammann S et al (2020) Extended hepatic metastasectomy for renal cell carcinoma-new aspects in times of targeted therapy: a single-center experience over three decades. Langenbecks Arch Surg 405(1):97-106. https://doi.org/10.1007/s00423-019-01852-4

6. Dabestani S, Marconi L, Hofmann F et al (2014) Local treatments for metastases of renal cell carcinoma: a systematic review. Lancet Oncol 15:e549-561

7. Fikatas P, Klein F, Andreou A et al (2016) Longterm survival after surgical treatment of renal cell carcinoma metastasis within the pancreas. Anticancer Res 36:4273-4278

8. Grassi P, Doucet L, Giglione P et al (2016) Clinica impact of pancreatic metastases from renal cell carcinoma: a multicenter retrospective analysis. PLoSONE 11:e151662

9. Hatzaras I, Gleisner AL, Pulitano C et al (2012) A multi-institution analysis of outcomes of liverdirected surgery for metastatic renal cell cancer. HPB (Oxford) 14:532-538

10. Hau HM, Thalmann F, Lubbert $C$ et al (2016) The value of hepatic resection in metastasic renal cancer in the era of tyrosinkinase inhibitor therapy. BMC Surg 16:49

11. Langan RC, Ripley RT, Davis JL et al (2012) Liver directed therapy for renal cell carcinoma. J Cancer 3:184-190

12. Ljungberg $B$, Bensalah $K$, Canfield Setal (2015) EAU guidelines on renal cell carcinoma: 2014 update. Eur Urol 67:913-924

13. Madkhali AA, Shin SH, Song KB et al (2018) Pancreatectomy for a secondary metastasis to the pancreas: a single-institution experience. Medicine (Baltimore) 97:e12653

14. Marchioni M, Bandini M, Pompe RS et al (2017) Survival of metastatic renal cell carcinoma patients continues to improve over time, even in targeted therapy era. Int Urol Nephrol 49:2143-2149

15. Pinotti E, Montuori M, Giani A et al (2019) Surgical treatment of liver metastases from kidney cancer: a systematic review. ANZJ Surg 89:32-37

16. Ruys AT, Tanis PJ, Nagtegaal ID et al (2011) Surgical treatment of renal cell cancer liver metastases: a population-based study. Ann Surg Oncol 18:1932-1938

17. Santoni M, Conti A, Partelli S et al (2015) Surgical resection does not improve survival in patients with renal metastases to the pancreas in the era of tyrosine kinase inhibitors. Ann Surg Oncol 22:2094-2100

18. Schwarz L, Sauvanet A, Regenet N et al (2014) Long-term survival after pancreatic resection for renal cell carcinoma metastasis. Ann Surg Oncol 21:4007-4013

19. Sperti C, Moletta L, Patane G (2014) Metastatic tumors to the pancreas: the role of surgery. World Gastrointest Oncol 6:381-392

20. Staehler MD, Kruse J, Haseke N et al (2010) Liver resection for metastatic disease prolongs survival in renal cell carcinoma: 12-year results from a retrospective comparative analysis. World J Urol 28:543-547

21. Strobel O, Buchler MW (2015) Pancreatic metastases from tumors in the urogenital tract. Gastrointest Tumors 2:75-82

22. Tanis PJ, van der Gaag NA, Busch OR et al (2009) Systematic review of pancreatic surgery for metastatic renal cell carcinoma. Br J Surg 96:579-592

23. Thelen A, Jonas S, Benckert C et al (2007) Liver resection for metastases from renal cell carcinoma. World J Surg 31:802-807

24. Tosoian JJ, Cameron JL, Allaf ME et al (2014) Resection of isolated renal cell carcinoma metastases of the pancreas: outcomes from the Johns Hopkins Hospital. J Gastrointest Surg 18:542-548

25. Wahba R, Stippel D, Bruns C (2018) Role of visceral surgery in oligometastases of non-gastrointestina tumors. Chirurg 89:523-528

26. Wente MN, Kleeff J, Esposito I et al (2005) Renal cancer cell metastasis into the pancreas: a singlecenter experience and overview of the literature. Pancreas 30:218-222

27. Yazbek T, Gayet B (2012) The place of enucleation and enucleo-resection in the treatment of pancreatic metastasis of renal cell carcinoma. JOP 13:433-438

28. Yu X, Wang B, Li X et al (2015) The significance of metastasectomy in patients with metastatic renal cell carcinoma in the era of targeted therapy. Biomed Res Int 2015:176373

29. Zaid HB, ParkerWP, Safdar NS et al (2017) Outcomes following complete surgical metastasectomy for patients with metastatic renal cell carcinoma: a systematic review and meta-analysis. J Urol 197:44-49

30. Zerbi A, Ortolano E, Balzano G et al (2008) Pancreatic metastasis from renal cell carcinoma: which patients benefit from surgical resection? Ann Surg Oncol 15:1161-1168 